Rheumatoid arthritis

\section{Safe and sound}

JD Mountz, J Chen and H-C Hsu

Gene Therapy (2005) 12, 1542-1543. doi:10.1038/sj.gt.3302606;

published online 25 August 2005

A newly published phase I clinical trial of a gene therapy approach to treating rheumatoid arthritis (RA) indicates that therapeutic genes can be transferred safely to, and be expressed in, rheumatoid joints. ${ }^{1}$

RA is a long-term, disabling disease that can lead to severe joint damage. The current biological therapies of choice for RA, such as administering systemic IL-1 receptor antagonist (IL-1Ra), have some major disadvantages, particularly the need to be regularly readministered. $^{2,3}$ Therefore, it would be highly advantageous to develop gene therapy approaches for stable and effective long-term delivery of such biological therapies to RA. Transplantation of genetically modified cells into the inflamed joint has been an approach to therapy for RA that has been explored for over a decade. During this time, there have been particularly encouraging results with the use of IL-1Ra-expressing cells in rabbits, ${ }^{4}$ mice $^{5}$ and rats. ${ }^{6}$ Based on these successes and the apparent lack of toxicity in preclinical trials, Evans et $a l^{1}$ carried out a phase I clinical trial using autologous rheumatoid arthritis synovial fibroblasts (RASF) transfected with IL$1 \mathrm{Ra}$ retrovirus.

This gene therapy approach has several advantages. ${ }^{1}$ First, transfer of injected cells greatly reduces or eliminates the possibility of germline transmission due to unwanted transfection of germ cells (Figure 1). Second, the gene therapy is delivered ex vivo into the autologous RASF harvested from the patient to receive such therapy, allowing simultaneous analysis of the cells ex vivo for high transduction and secretions of the gene therapy as well as access of the down-stream biological effects. This suggests that, with this combined autologous cell gene therapy and in vitro studies approach, it is possible to enable the gene therapy to be tailored to best inhibit proinflammatory, synovial hyperprolifera-

\section{In vitro retrovirus \\ IL-1Ra gene transfer}

tive, or bone erosive properties of each patient.

However, the key goal of the new study was to assess the safety of the ex vivo gene therapy approach: an issue of paramount importance for its application in humans.

One safety feature of this phase I safety trial is that the metacarpophalangeal (MCP) joints that were receiving therapy were resected for implant replacement treatment 1 week later, and the transduced cells were found to be localized at the surface of the synovium, the synovial lining. This is an ideal location for the therapeutic cells, since production of the therapeutic molecule at this site of most active disease should lead to the best therapy with the lowest systemic side effects. It is also highly commendable that these investigators have waited an additional 5 years before reporting the results of this phase I safety study after which time no patient has developed evidence of transformed cells elsewhere.

The one potentially worrying result in this study came from the patient that received the highest number of transgenic cells ( $10^{7}$ cells). In this patient, cells that expressed IL-1Ra were found in an adjacent control joint. It would be important to determine if this was due to cross contamination at the time of injection or at the time of surgical resection, or if it was due to migration of cells from one joint to the other after injection. In future trials, it will be possible to insert a suicide gene such as thymidine kinase into the cell that can be activated with ganciclovir to kill transfected cells should they oversecrete or become transformed, which should provide an additional level of safety. ${ }^{7}$

From a practical standpoint for local therapy, one should consider how many joints can be treated with the approach described by Evans et al. ${ }^{1}$ The authors admit that the autologous cell gene therapy is costly, labor intensive, and time consuming. However, RA affects primarily the small joints of the hands and feet. It is conceivable that if a single dose can provide longterm therapy, and if cell storage and transfection techniques can be made more cost efficient, then this therapy may be applicable especially to RA
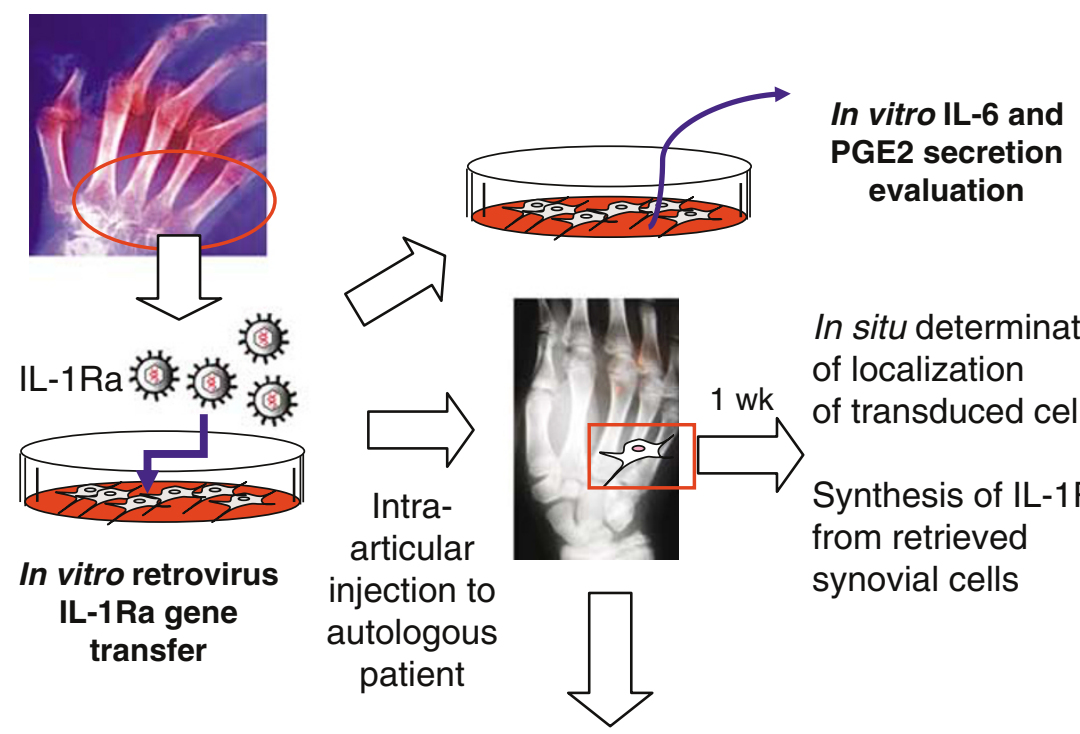

In situ determination of localization of transduced cells Synthesis of IL-1Ra from retrieved synovial cells

5 yr long-term safety outcome evaluation

Figure 1 Schematic diagram of the combined ex vivo safety tests and in vivo autologous cell gene therapy for rheumatoid arthritis (The joint pictures are adapted with permission from the UAB Arthritis and Musculoskeletal Center website at www.uab.edu). 
patients with limited disease affecting a smaller number of joints.

Somatic gene transfer is a promising therapeutic strategy, but it may also evoke new types of side effects related to genetic damage or transgene activity. The beauty of cell gene therapy approaches is that they may allow the genetically engineered cells to be studied in vitro before transfer into the patient to ensure that there is no abnormally suggestive transformation of cells or DNA damage. It is known that such changes may only become apparent much later after in vivo transfer as with severe combined immune deficiency. Gene therapy was carried out using the same viral vector backbone, MFG, as used in this study, in which 3/11 children developed leukemia due to the retroviral integrations in the putative oncogene LMO2. ${ }^{8}$ If more was known about the factors that regulate retrovirus integration, and how this might lead to transformation in cells, it may be possible to develop methods for more specific DNA targeting to prevent such tragic outcomes or at least warrant more ex vivo safety trials before the clinical trials are carried out. In the future, advances in stem cell technology and therapies may make it possible to have stem cells from a patient to be differentiated into different cells for safe genetic modification and reimplantation for highest local effectiveness and lowest risk.

In addition to safety, a biosensing system with an inducible transcriptional switch that allows robust but adjustable transgene expression is required. ${ }^{9}$ Inflammation-inducible promoters are likely candidates to achieve precise control of transgene expression by physiologically driven processes. With such promoters, gene therapy could enter a new era of temporal control of the therapeutic transgene expression. The ideal expression system should contain an amplification loop for high transgene expression and a drug-controllable switch to allow intervention by the physician. The merging of these modalities may provide a flexible system to fine-tune transgene expression, which is a prerequisite for the implementation of gene therapy in RA.

JD Mountz, J Chen and $\mathrm{H}-\mathrm{C} \mathrm{Hsu}$ are at the Division of Clinical Immunology and Rheumatology, Department of Medicine, University of Alabama at Birmingham, and Birmingham Veterans Administration Medical Center, 701 S. 19th Street, Room 473, Birmingham, $A L$ 35294, USA.

E-mail:John.Mountz@ccc.uab.edu

Published online 25 August 2005

1 Evans $\mathrm{CH}$ et al. Gene transfer to human joints: progress toward a gene therapy of arthritis. Proc Natl Acad Sci USA 2005; 102: 8698-8703.
2 Bresnihan B. The safety and efficacy of interleukin-1 receptor antagonist in the treatment of rheumatoid arthritis. Semin Arthritis Rheum 2001; 30: 17-20.

3 Nuki G, Bresnihan B, Bear MB, McCabe D. Long-term safety and maintenance of clinical improvement following treatment with anakinra (recombinant human interleukin-1 receptor antagonist) in patients with rheumatoid arthritis: extension phase of a randomized, double-blind, placebo-controlled trial. Arthritis Rheum 2002; 46: 2838-2846.

$4 \mathrm{Kim} \mathrm{SH}$ et al. Ex vivo gene delivery of IL-1Ra and soluble TNF receptor confers a distal synergistic therapeutic effect in antigen-induced arthritis. Mol Ther 2002; 6: 591-600.

5 Smeets RL et al. Effectiveness of the soluble form of the interleukin-1 receptor accessory protein as an inhibitor of interleukin-1 in collagen-induced arthritis. Arthritis Rheum 2003; 48: 2949-2958.

6 Makarov SS et al. Suppression of experimental arthritis by gene transfer of interleukin 1 receptor antagonist cDNA. Proc Natl Acad Sci USA 1996; 93. 402-406.

7 Sant SM et al. Molecular lysis of synovial lining cells by in vivo herpes simplex virus-thymidine kinase gene transfer. Hum Gene Ther 1998; 9: 2735-2743.

8 Hacein-Bey-Abina $\mathrm{S}$ et al. LMO2-associated clonal $\mathrm{T}$ cell proliferation in two patients after gene therapy for SCID-X1. Science 2003; 302: 415-419.

9 Vilaboa $\mathrm{N}$ et al. Novel gene switches for targeted and timed expression of proteins of interest. Mol Ther 2005; 12: 290-298. 